\title{
Analysis of a Rectangular Slot in the Ground Plane of a Stripline with Uniaxial Substrates
}

\author{
A. V. Proaño De la Torre ${ }^{1^{*}}$, J. C. da S. Lacava ${ }^{2}$, and L. Cividanes ${ }^{3}$ \\ ${ }^{1}$ Army Polytechnic School, ESPE, Sangolqui, Ecuador \\ ${ }^{2}$ Department of Electronics Engineering, ITA, S. J. Campos, 12228-900 Brazil \\ ${ }^{3}$ Brazilian Institute for Space Research, INPE, S. J. Campos, I2001-970 Brazil
}

\begin{abstract}
1. Introduction
Printed aperture in the ground plane of a microstrip line is a versatile radiating element. The main advantages of radiating apertures are wider bandwidth, less interaction via surface waves, better isolation and negligible direct radiation from the feed network [1]. However, a strong backlobe radiation due to the resonant aperture is one disadvantage. In order to avoid this undesirable effect, one possibility is the utilization of a stripline structure to feed the aperture. On the other hand, many materials commonly used as substrates for microstrip antennas exhibit a significant amount of anisotropy, specially the uniaxial one [2]. As a consequence, their electromagnetic parameters can be affected by a non negligible quantity. In particular, it is well-known the influence of the uniaxial anisotropy on the resonant frequency of these antennas [3-5]. In this paper, using the full-wave spectral-domain approach and the method of moments, a rectangular slot in the ground plane of a stripline with uniaxial substrates is analyzed. Effects of the anisotropy on the input impedance of the antenna are presented. Results for the characteristic impedance and the propagation constant of the stripline will be discussed during the presentation.
\end{abstract}

\section{Theory}

The structure under consideration consists of a thin conducting strip located at the interface between two anisotropic layers, which are sandwiched by two ground planes. The rectangular slot is printed in the ground plane located on the $x-y$ plane of a rectangular coordinate system, with a protection layer covering it, as illustrated in Fig.1. In our formulation, each layer is considered as a linear homogeneous dielectric medium with uniaxial anisotropy, i.e., a medium described by the tensor permittivity $\widetilde{\varepsilon}_{n}$ (the index $n=1,2$ or 3 identifies each anisotropic layer) and by the permeability $\mu=\mu_{0}$. The planar interface $z=d_{3}$ separates the cover layer from the free space region $\left(z>d_{3}\right.$, permittivity $\varepsilon_{0}$, and permeability $\mu_{0}$ ). The theory used in this work considers the antenna structure as a boundary value problem where the surface electric current density on the conducting strip and the tangential electric field component in the aperture plane (slot) are the virtual sources of the electromagnetic fields. Starting from Maxwell's equations, the wave equations in the anisotropic layers and in the free space region are determined. In our approach, the wave equations are solved in the Fourier domain and the boundary conditions for the 


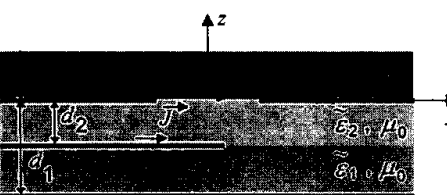

Fig. 1 - Geometry of a rectangular slot fed by an open end stripline.

electromagnetic fields are applied at the interfaces $z=0, z=-d_{1}, z=-d_{2}$, and $z=d_{3}$. Expressions for the spectral electromagnetic fields are determined by solving the resulting set of equations. As the calculation is carried out in the Fourier domain, the spectral Green functions can be obtained in compact and closed forms. Finally, to determine the fields in the space domain, the inverse Fourier transform is applied.

\section{Method of Moments}

The coupled integral equations can be obtained by enforcing the following boundary conditions: the total tangential magnetic field must be continuous across the rectangular slot and the total tangential electric field must vanish along the stripline surface. These integral equations are solved numerically by the well-known method of moments technique. In this approach, the electric current density on the stripline and the tangential electric field component in the aperture plane are expanded as a set of basis functions. Sinusoids were used to represent incident and reflected traveling waves of the fundamental stripline mode, and triangular subsectional functions were used near the open end Triangular subsectional functions were also used for the tangential electric field component in the aperture plane. As test functions only triangular subsectional modes (with edge conditions) were used. Solving the resulting linear system, tangential electric field component in the aperture plane, reflection coefficient and current distribution on the stripline can be obtained. After that, calculating the characteristic impedance $\left(Z_{0}\right)$ and the propagation constant of the stripline, the antenna input impedance can be determined.

\section{Numerical Results}

Fig. 2 shows the plant view of the rectangular slot. For brevity, only results for the antenna with dimensions shown in Fig. 3 are discussed below.

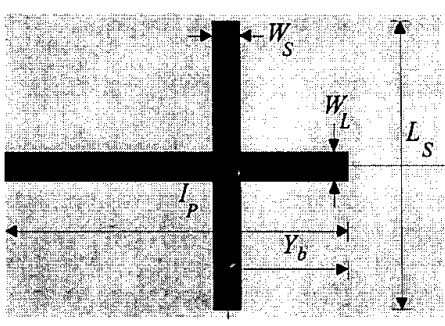

Fig. 2 - Plant view of a rectangular slot fed by

$\dot{y}$ an open end stripline.

$W_{S}$ is the slot width

$W_{L}$ is the stripline width 
Graphics for the normalized input impedance $z_{i n}$, calculated at $y=-\left(I_{p}-Y_{b}\right)$, are presented in Fig. 3. Increasing the anisotropic ratio $\left(\varepsilon_{r z} / \varepsilon_{r x}\right)$ the input impedance is affected by a non negligible quantity, while the resonant frequency decreases.

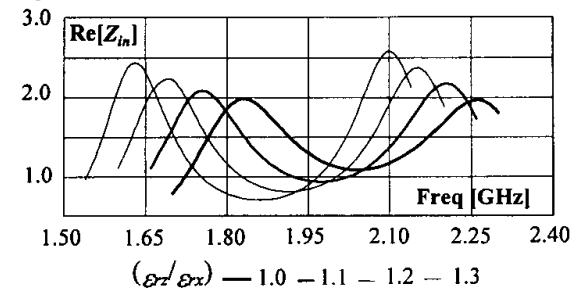

Layer 1: $\varepsilon_{r z l}=\varepsilon_{r x l}=1.0$

Layer 2: $\varepsilon_{r x 2}=2.55$

Loss tangent $=0.0018$

Layer 3: $\varepsilon_{r \times 3}=2.55$

Loss tangent $=0.0018$

$(g r z=1.0 x)-1.1-1.2-1.3$

$L_{S}=41.0 \mathrm{~mm}$

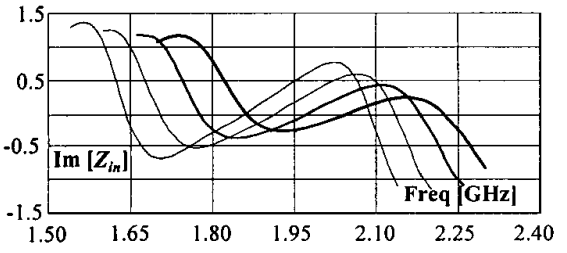

$W_{S}=4.0 \mathrm{~mm}$

$Y_{b}=8.8 \mathrm{~mm}$

$I_{p}=110.55 \mathrm{~mm}$

$W_{L}=5.6 \mathrm{~mm}$

$d_{1}=3.1524 \mathrm{~mm}$

$d_{2}=d_{3}=1.524 \mathrm{~mm}$

Fig. 3 - Normalized input impedance $z_{\text {in }}\left(z_{\text {in }}=Z_{\text {in }} / Z_{0}\right)$.

Results for the isotropic case $\left(\varepsilon_{r z 1}=\varepsilon_{r x l}=1.0, \varepsilon_{r z 2}=\varepsilon_{r x 2}=\varepsilon_{r 23}=\varepsilon_{r \times 3}=2.55\right)$, obtained directly from the uniaxial solution, are presented in Figs. 4-6. Normalized input impedance are plotted in Fig. 4. Good agreement between our calculation (MMAPP) and that obtained by using the Ensemble ${ }^{\mathrm{TM}}[6]$ is observed. Effects of the triangular basis function domain of the current density on the normalized input impedance were also analyzed. In case under consideration, the stability of the solution was obtained when the domain reached $164 \mathrm{~mm}$, as shown in Fig. 5.

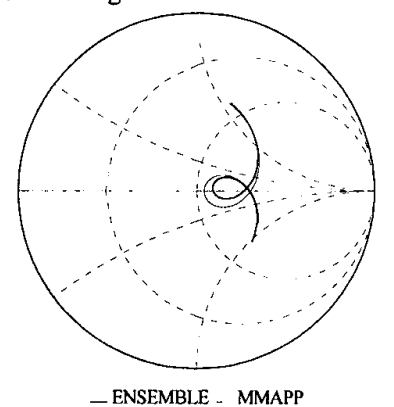

Fig. 4 - Normalized input impedance for isotropic case.

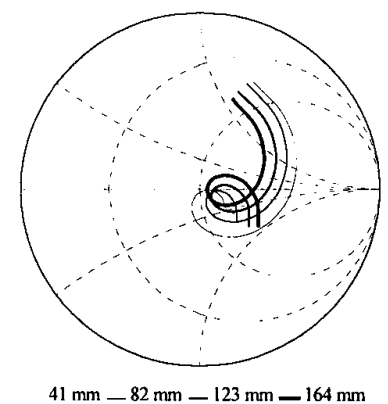

Fig. 5 - Effects of the triangular basis function domain. 
Finally, a 3-D graphic for the surface electric current density on the stripline is presented in Fig. 6.

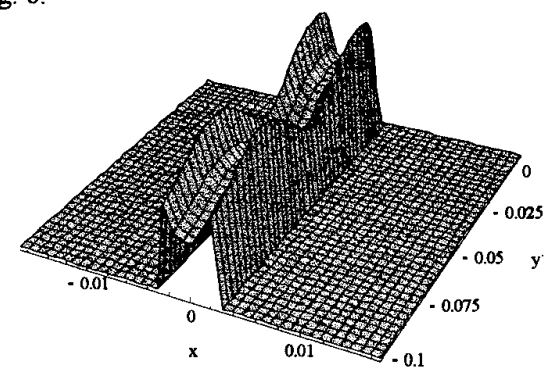

Fig. 6 - Surface electric current density on the stripline.

\section{Conclusions}

A rigorous theoretical formulation for analyzing a rectangular slot printed in the ground plane of a stripline with uniaxial substrate has been presented. Spectral Green functions in compact and closed forms have been derived. The effect of the anisotropic ratio $\left(\varepsilon_{r z} / \varepsilon_{r x}\right)$ on the normalized input impedance of the antenna has been studied. As a result of our calculation it is clear that increasing this anisotropic ratio the antenna input impedance is affected by a non negligible quantity, while the resonant frequency decreases.

Acknowledgment: This work was supported in part by CAPES and ICAOBRA/95/802, Objective 6 .

\section{References}

[1]M. Kahrizi, T. P. Sarkar, and Z. A. Mariecevic, "Analysis of a wide radiating slot in the ground plane of a microstrip line," IEEE Trans. Microwave Theory Tech., vol. 41, pp. 29-37, Jan. 1993.

[2]N. G. Alexópoulos, "Integrated-circuit structures on anisotropic substrates." IEEE Trans. Microwave Theory Tech., vol. 33, pp. 847-881, Oct. 1985.

[3]D. M. Pozar, "Radiation and scattering from a microstrip patch on a uniaxial substrate," IEEE Trans. Antenmas Propagat., vol. 35, pp. 613-621, June 1987.

[4]R. M. Nelson, D. A. Rogers, and A. G. D'Assunção, "Resonant frequency of a rectangular microstrip patch on several uniaxial substrate," IEEE Trans. Antennas Propagat., vol. 38, pp. 973-981, July 1990.

[5]F. Bouttout, F. Benabdelaziz, A. Benghalia, D. Khedrouche, and T. Fortaki, "Uniaxially anisotropic substrate effects on resonance of rectangular microstrip patch antenna," Electronics Letters, vol. 35, pp. 255-256, Feb. 1999.

[6]Ensemble: Design, Review, \& 1D Array Synthesis, Version 4.1a, Boulder Microwave Technologies, Inc 